\title{
HESS J1632-478: an energetic relic
}

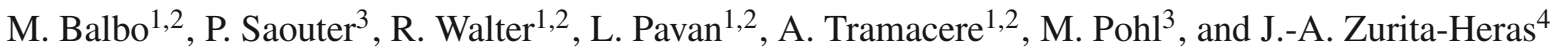 \\ 1 INTEGRAL Science Data Centre, Université de Genève, Chemin d'Ecogia 16, 1290 Versoix, Switzerland \\ e-mail: Matteo.Balbo@unige.ch \\ 2 Observatoire de Genève, Université de Genève, Chemin des Maillettes 51, 1290 Sauverny, Switzerland \\ 3 Département de Physique Nucléaire et Corpusculaire, Université de Genève, Quai Ernest-Ansermet 24, 1211 Genève 4, Switzerland \\ 4 AIM Paris Saclay, CEA/DSM-CNRS/INSU-Université Paris Diderot, IRFU/Service d'Astrophysique, 91191 Gif-sur-Yvette, France
}

Received 11 April 2010 / Accepted 4 July 2010

\section{ABSTRACT}

\begin{abstract}
Aims. HESS J1632-478 is an extended and still unidentified TeV source in the galactic plane.
Methods. In order to identify the source of the very high energy emission and to constrain its spectral energy distribution, we used a deep observation of the field obtained with XMM-Newton together with data from Molonglo, Spitzer and Fermi to detect counterparts at other wavelengths.

Results. The flux density emitted by HESS J1632-478 peaks at very high energies and is more than 20 times weaker at all other wavelengths probed. The source spectrum features two large prominent bumps with the synchrotron emission peaking in the ultraviolet and the external inverse Compton emission peaking in the TeV. HESS J1632-478 is an energetic pulsar wind nebula with an age of the order of $10^{4}$ years. Its bolometric (mostly GeV-TeV) luminosity reaches $10 \%$ of the current pulsar spin down power. The synchrotron nebula has a size of $1 \mathrm{pc}$ and contains an unresolved point-like X-ray source, probably the pulsar with its wind termination shock.
\end{abstract}

Key words. acceleration of particles - stars: neutron - gamma rays: stars - X-rays: stars - pulsar: general

\section{Introduction}

HESS J1632-478 has been discovered as a diffuse very high energy (VHE) $\gamma$-ray source during the 2004-2006 survey of the inner Galaxy (Aharonian et al. 2006a) performed with the High Energy Stereoscopic System Cherenkov Telescope Array (HESS; Hinton 2004).

Though many galactic VHE sources are recognized to be supernova remnants, pulsars and pulsar wind nebulae, a small number of them still lack a clear identification. HESS J1632478 is one of them, even if tentatives to explain its nature have already been presented (Aharonian et al. 2006a; Walter 2007).

A detailed VHE spectral and positional analysis of HESS J1632-478 has been reported in Aharonian et al. (2006a), based on $4.5 \mathrm{~h}$ of HESS observations. The source best fit position is $\mathrm{RA}=16: 32: 09 \mathrm{Dec}=-47: 49: 12(\mathrm{~J} 2000)$, placing it in the direction of the near $3 \mathrm{kpc}$ arm tangent in the galactic plane.

The $\mathrm{TeV}$ source is extended with a semi-major axis of $(12 \pm 3)^{\prime}$, forming an angle of $21 \pm 13^{\circ}$ with respect to the positive galactic longitude axis. The VHE spectrum, between 0.2 and $4.5 \mathrm{TeV}$, can be fitted with a powerlaw model, yielding a photon index $\Gamma=2.12 \pm 0.20$ and a flux above $200 \mathrm{GeV}$ of $(28.7 \pm 5.3) \times 10^{-12} \mathrm{ph} \mathrm{cm}^{-2} \mathrm{~s}^{-1}$.

To understand HESS J1632-478, we collected multiwavelength data from Fermi, XMM-Newton, Spitzer and Molonglo, to construct its spectral energy distribution and discuss the emission mechanisms.

\section{X-ray counterpart}

\subsection{XMM-Newton observation and data analysis}

The XMM-Newton observatory (Jansen et al. 2001) includes three $1500 \mathrm{~cm}^{2} \mathrm{X}$-ray telescopes each with a European Photon
Imaging Camera (EPIC) at the focus. Two of the EPIC imaging spectrometers use MOS CCDs (Turner et al. 2001) and one uses pn CCDs (Strüder et al. 2001).

In the period from August to September 2008, XMM-Newton performed 9 observations of a field centered close to HESS J1632-478, collecting data for a total of $92 \mathrm{ks}$. The EPIC observations, listed in Table 1, used the thin optical blocking filter. The EPIC pn camera was operated in large-window mode.

Two other XMM-Newton observations of the same field were collected in 2003 and 2004. As these observations used the medium optical blocking filter and did not use the central MOS CCD and the 2004 one was heavily affected by an outburst of 4 U 1630-472, we did not combine these observations with those of 2008 .

Standard data reduction procedures were applied to each observation using the XMM SCIENCE ANALYSIS SOFTWARE (SAS Version 9.0.0) ${ }^{1}$. Source positions were derived using the SAS task edetect_chain with the EPIC MOS and pn data. Only well calibrated single pixel events were selected for the pn CCD and single and double events for the MOS CCDs. Known hot, or flickering, pixels and electronic noise were rejected. Events were further screened with the conservative FLAG $=0$ criteria and selected for the energy ranges $(0.2-12 \mathrm{keV}$ for MOS and $0.2-15 \mathrm{keV}$ for $\mathrm{pn})$. Periods with enhanced background - soft proton flares (EPIC pn count rate above $10 \mathrm{keV}$ larger than 0.7 to $4.5 \mathrm{ct} / \mathrm{s}$, depending on the observation) - were disregarded in the analysis, resulting in total filtered exposures of $87 \mathrm{ks}$ and $62 \mathrm{ks}$ for the MOS and pn cameras respectively.

Events were finally selected in the source and background regions. Circular regions have been chosen for point sources.

\footnotetext{
1 http://xmm. esac. esa.int/sas/current/
} 
Table 1. Observation identifiers and date for all XMM-Newton observations of 2008 including the source HESS J1632-478.

\begin{tabular}{ccrrrr}
\hline \hline Obs. ID & Date & $\begin{array}{c}\text { MOS } \\
\text { act. s }\end{array}$ & $\begin{array}{r}\text { MOS } \\
\text { filt. s }\end{array}$ & $\begin{array}{c}\text { pn } \\
\text { act. s }\end{array}$ & $\begin{array}{c}\text { pn } \\
\text { filt. s }\end{array}$ \\
\hline 556140101 & 14.08 .08 & 10621 & 10621 & 9834 & 5800 \\
556140201 & 16.08 .08 & 9620 & 7500 & 8034 & 5900 \\
556140301 & 18.08 .08 & 8619 & 7500 & 7034 & 4500 \\
556140401 & 20.08 .08 & 12829 & 12821 & 11234 & 11000 \\
556140501 & 21.08 .08 & 4951 & 4951 & 2239 & 1900 \\
556140601 & 22.08 .08 & 14027 & 14027 & 12398 & 12398 \\
556140701 & 24.08 .08 & 10621 & 10621 & 9834 & 6700 \\
556140801 & 26.08 .08 & 11219 & 11219 & 9634 & 9500 \\
556141001 & 17.09 .08 & 9118 & 8200 & 7534 & 4500 \\
\hline
\end{tabular}

Notes. The actual and filtered exposure times in seconds are given for the two EPIC MOS and for the pn camera respectively.

Table 2. Source identifier, name, positions and positional statistical error $(\Delta)$ derived from $X M M$-Newton data.

\begin{tabular}{llccc}
\hline \hline ID & Name & RA & Dec & $\Delta$ \\
\hline A & XMMU J163219.9-474731 & $16: 32: 19.9$ & $-47: 47: 31$ & $0.19^{\prime \prime}$ \\
B & AX J1632.8-4746 & $16: 32: 48.2$ & $-47: 45: 04$ & $0.13^{\prime \prime}$ \\
C & IGR J16320-4751 & $16: 32: 01.9$ & $-47: 52: 29$ & $0.87^{\prime \prime}$ \\
Ee & HESS J1632-478 & $16: 32: 07.8$ & $-47: 49: 09$ & $1^{\prime \prime}$ \\
Ep & HESS J1632-478 & $16: 32: 08.8$ & $-47: 49: 01$ & $0.5^{\prime \prime}$ \\
\hline
\end{tabular}

Notes. The systematic error on the XMM-Newton attitude reconstruction is of the order of $1.5^{\prime \prime}$.

The regions used for the likely counterpart to HESS J1632-478 are described in Sect. 2.3.

\subsection{Serendipitous sources}

In the nine XMM-Newton observations of 2008 , three pointlike sources are always clearly detected: IGR J16320-4751, AX J1632.8-4746 and XMMU J163219.9-474731, by order of decreasing X-ray flux. The EPIC derived positions of these sources are listed in Table $2^{2}$.

The XMM-Newton data of the high-mass X-ray binary IGR J16320-4751 will be presented elsewhere. In these data, IGR J16320-4751 features column densities in the range $(8-10) \times 10^{22} \mathrm{~cm}^{-2}$.

AX J1632.8-4746 is detected by XMM-Newton as a constant source with $(14.5 \pm 0.3) \times 10^{-2}$ counts s$^{-1}$. Its spectrum can be well reproduced with the absorbed emission of a hot diffuse gas (mekal, Kaastra 1992) yielding $N_{\mathrm{H}}=(6.2 \pm 0.2) \times$ $10^{22} \mathrm{~cm}^{-2}, k T=2.5 \pm 0.12 \mathrm{keV}$ and $F_{2-10 \mathrm{keV}}=(3.25 \pm$ $0.1) \times 10^{-12} \mathrm{erg} \mathrm{cm}^{-2} \mathrm{~s}^{-1}$. The ASCA data (Sugizaki et al. 2001) were described with an absorbed power-law resulting in a folded model comparable to that derived with XMM-Newton.

The light-curve of XMMU J163219.9-474731 does not show any variation. Its spectrum is also well reproduced with an absorbed mekal model with $N_{\mathrm{H}}=(5.5 \pm 0.3) \times 10^{22} \mathrm{~cm}^{-2}, k T=$ $2.2 \pm 0.1 \mathrm{keV}$ and $F_{2-10 \mathrm{keV}}=(1.1 \pm 0.1) \times 10^{-12} \mathrm{erg} \mathrm{cm}^{-2} \mathrm{~s}^{-1}$.

The spectra of both AX J1632.8-4746 and XMMU J163219.9-474731 are consistent with these of massive stars. If these sources are at a distance $\gtrsim 2 \mathrm{kpc}$, their $\mathrm{X}$-ray luminosity $\left(\$ 10^{33} \mathrm{erg} / \mathrm{s}\right)$ is consistent with the expectations $\left(10^{30-33} \mathrm{erg} / \mathrm{s}\right.$, Cassinelli et al. 1981). We find possible

\footnotetext{
${ }^{2}$ http://xmm.vilspa.esa.es/docs/documents/ CAL-TN-QQ18.pdf
}

infrared point-like counterparts to these two XMM sources in the Spitzer surveys (Benjamin et al. 2003; Carey et al. 2009) at distances of $3.3^{\prime \prime}$ and $2.0^{\prime \prime}$ respectively.

\subsection{HESS J1632-478 counterpart}

None of the point-like X-ray sources discussed above is a likely counterpart of the extended source detected in the HESS survey, therefore we merged together all the observations to obtain a better sensitivity. To construct mosaic images of the MOS and pn data, described in Sect. 2.1, we used the emosaic command. The mosaic of the count images has been divided by the mosaic of the individual exposure maps calculated with the eexmap command.

The resulting MOS and PN mosaic images are presented in Fig. 1. An extended source is detected in the two mosaic images (source E). The source profile features an unresolved point-like component (source Ep in Table 2), with a width $(\sigma=6.8 \pm$ $0.6)^{\prime \prime}$ consistent with the XMM-Newton point spread function, in addition to an extended component (source Ee in Table 2), close to the detection limit. The point and extended sources have significances of 15 and $18 \sigma$ respectively. The extended source could be represented by a two dimensional Gaussian profile with $1 \sigma$ semi-axes of $(32.0 \pm 1.2)^{\prime \prime}$ and $(15.3 \pm 0.7)^{\prime \prime}$. The angle of the major axis of the extended source is $(55 \pm 2)^{\circ}$ counted anticlockwise from the north direction.

To extract the X-ray spectra of the point-like and extended source, we first defined the source (Fig. 1 bottom) and background (Fig. 1 top and middle) extraction regions in the mosaic images and selected the events in these regions for each individual observation. The elliptical region used to select the extended source excludes a circle around the point-like source (Fig. 1 bottom). The ancillary spectral response file for the extended source was generated with the extendedsource=YES option of the arfgen tool.

For the MOS CCDs, the background extraction region was chosen in order to minimize the possible contributions from IGR J16320-4751 and XMMU J163219.9-474731 (Fig. 1 top). A different background extraction region was chosen for the PN camera (Fig. 1 middle) to avoid falling into the gap between the CCD chips when extracting the background in individual observations.

We applied these extraction regions to each observation separately. Source and background spectra and response matrices were extracted for each observation. As the source is at the same position in the detector plane for all the 2008 observations, the resulting spectra were combined together using addspec (without error propagation, i.e. using Poisson statistics) to obtain a single merged spectrum for each camera.

The resulting spectra have been analyzed with XSPEC (version 11$)^{3}$. We fitted simultaneously the merged EPIC spectra obtained for the three cameras with an absorbed power-law model (Fig. 2). The resulting $\chi_{v}^{2}$ (Table 3) are large, probably because the count rate uncertainties related to the background subtraction are underestimated.

The absorbed and unabsorbed fluxes of the extended source are $F_{2-10 \mathrm{keV}}=1.4 \times 10^{-13} \mathrm{erg} \mathrm{cm}^{-2} \mathrm{~s}^{-1}$ and $4.3 \times$ $10^{-13} \mathrm{erg} \mathrm{cm}^{-2} \mathrm{~s}^{-1}$, respectively.

We also attempted to fit the point-like source with an absorbed black-body model. The derived temperature and emitting radius are $1 \mathrm{keV}$ and $22-43 \mathrm{~m}$ (for a distance of $3 \mathrm{kpc}$ ). These parameters are not meaningful as a power-law model fits the data

\footnotetext{
${ }^{3}$ http://heasarc . nasa.gov/docs/xanadu/xspec/xspec11/
} 


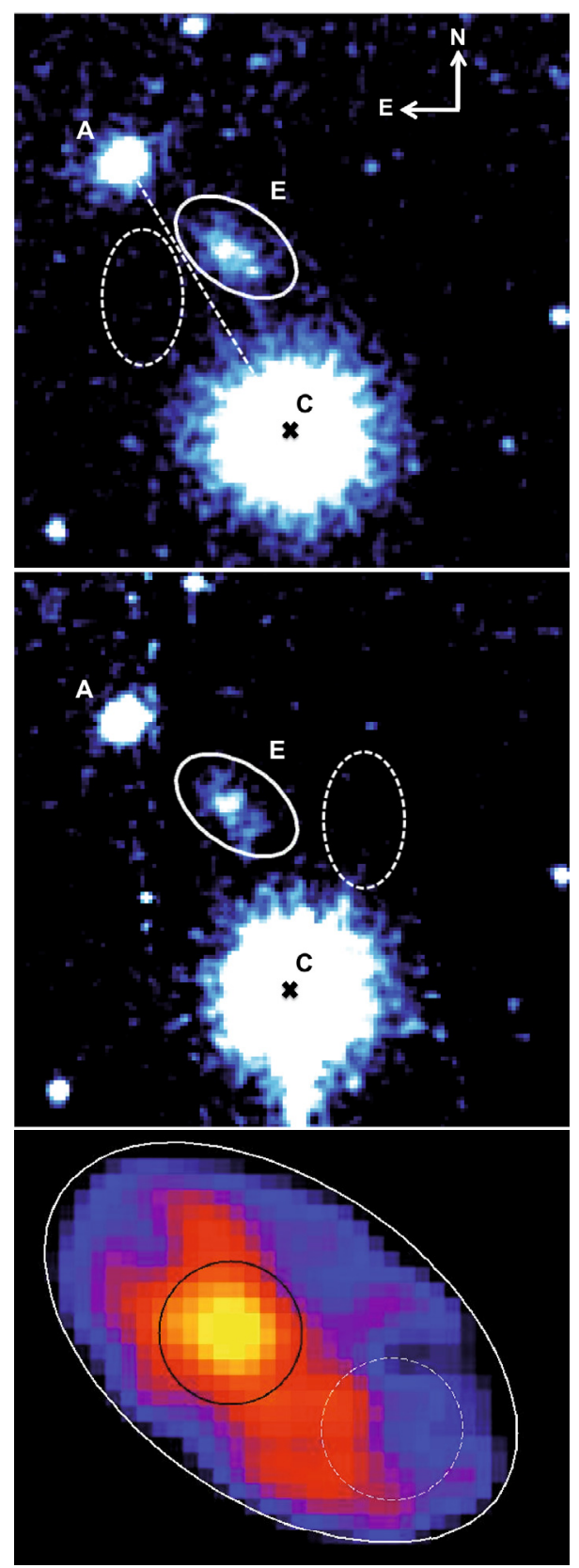

Fig. 1. The upper and middle panels show XMM-Newton EPIC mosaic images in the energy band $0.2-12 \mathrm{keV}$ for the MOS and $0.2-15 \mathrm{keV}$ for pn CCDs, respectively. A smoothing algorithm was applied. The height of these images is 10 arcmin. Ellipses indicate the source (continuous) and background (dashed) extraction regions for the extended source. The enlarged bottom image extracted from the MOS1 camera, shows the extraction regions for the point-like (black) and extended (white) sources respectively. Events in the black circle are not included in the spectrum of the extended source. The dashed white circle shows the region used to select the "background" events of the point-like source.

as well and is probably a better representation of the emission at the termination shock.

\section{Radio counterpart}

The MGPS-2 is a high-resolution and large-scale survey of the galactic plane carried with the Molonglo Observatory Synthesis Telescope (MOST) at a frequency of $843 \mathrm{MHz}$ (Murphy et al. 2007). Close to the position of the extended X-ray source, the
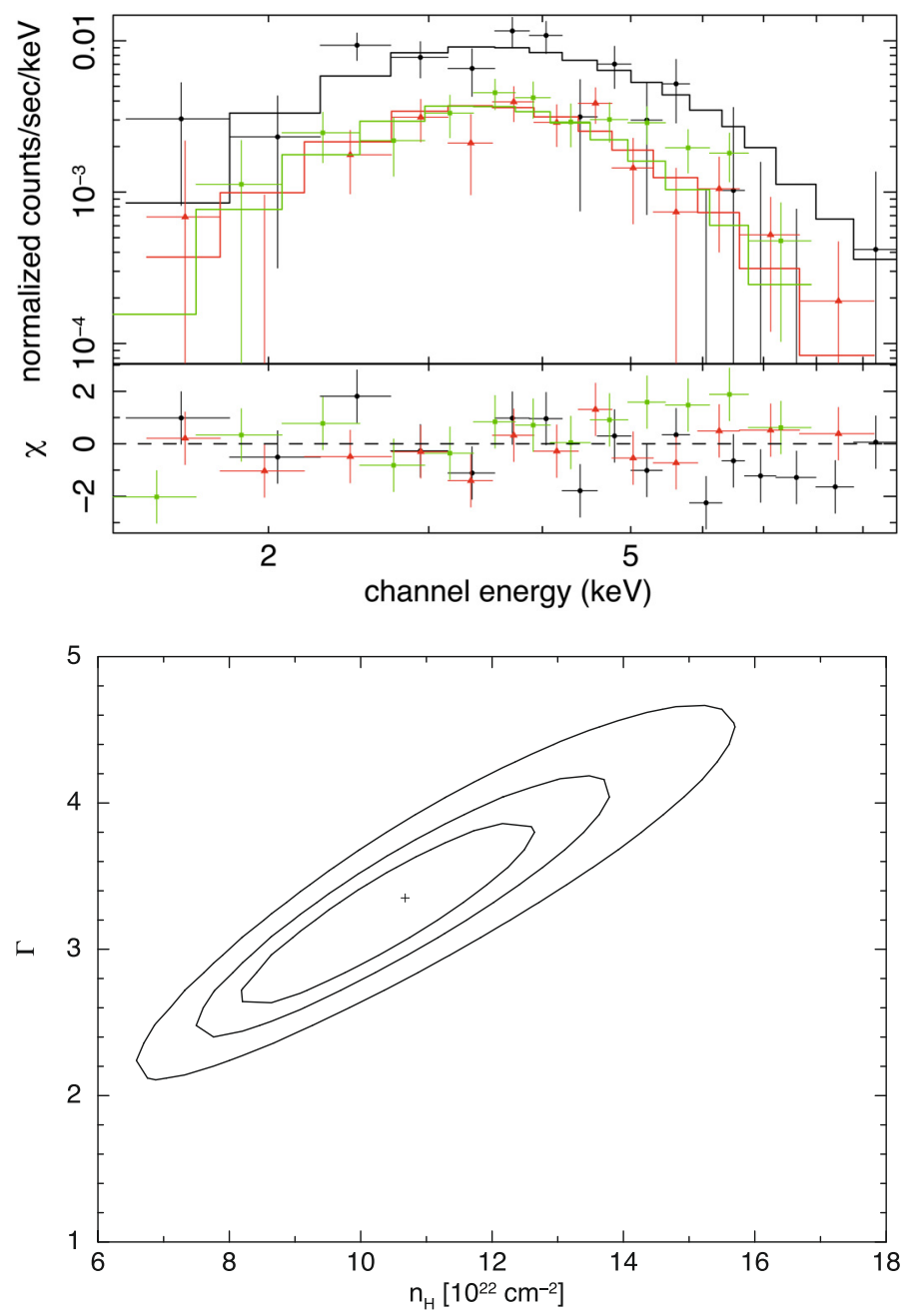

Fig. 2. Top: best power-law fit of the spectra of the extended X-ray source obtained from the three EPIC cameras. Bottom: 68\%, $90 \%$ and $99 \%$ confidence contours for the $N_{\mathrm{H}}-\Gamma$ parameters.

Table 3. Best fit parameters for an absorbed power-law model fitted simultaneously to the three EPIC spectra on the point-like and extended sources.

\begin{tabular}{cccl}
\hline \hline Parameter & Point & Extended & Unit \\
\hline$N_{\mathrm{H}}$ & $13_{-4}^{+6}$ & $11_{-2.7}^{+2.2}$ & $10^{22} \mathrm{~cm}^{-2}$ \\
$\Gamma$ & $2.6_{-0.8}^{+1.3}$ & $3.4_{-0.8}^{+0.6}$ & \\
$F_{2-10 \mathrm{keV}}$ & $2.3_{-1.0}^{+0.3}$ & $4.3_{-0.4}^{+0.8}$ & $10^{-13} \mathrm{erg} \mathrm{cm}^{-2} \mathrm{~s}^{-1}$ \\
$\chi_{v}^{2}$ & $1.8^{2}$ & 1.9 & \\
\hline
\end{tabular}

Notes. Reported errors correspond to $1 \sigma$ uncertainties $(68 \%)$. The 2-10 keV fluxes are corrected for the absorption.

root-mean-square sensitivity is $1-2 \mathrm{mJy} /$ beam and the beam size has a $1 \sigma$ semi-axis of $20^{\prime \prime}$ (obtained by fitting a nearby point-like source).

Figure 3 displays the MGPS-2 image of the region around HESS J1632-478. The extended XMM-Newton source lies in a large negative area, caused by artifacts of the image reconstruction. An extended diffuse radio source, with a size corresponding very well to the XMM-Newton diffuse counterpart (the white ellipse in Fig. 3) is clearly observed. By comparing this source with the surrounding region, a positive excess of the order of $\sim 16 \mathrm{mJy} /$ beam, can be extracted, whereas the distribution of the counts in the $4^{\circ} \times 4^{\circ}$ image has a $1 \sigma$ width smaller 


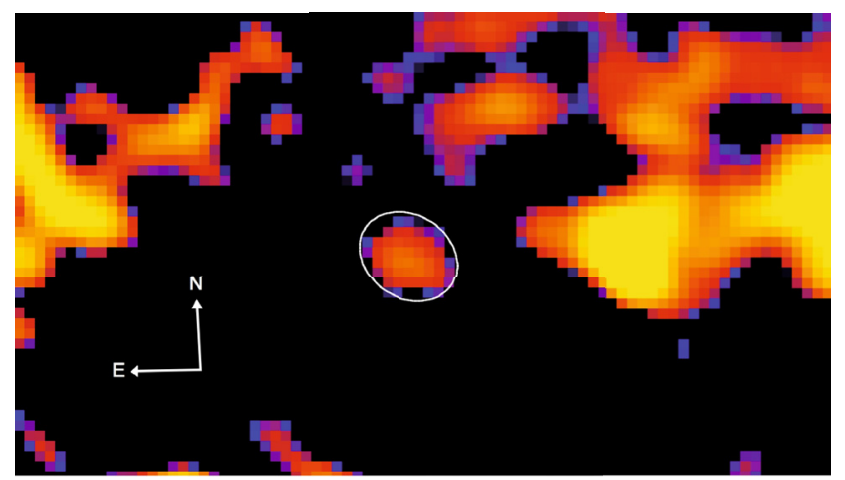

Fig. 3. Image $\left(15^{\prime} \times 11^{\prime}\right)$ from the second epoch Molonglo Galactic Plane Survey (MGPS-2) at $843 \mathrm{MHz}$. Colors are in square root scale between -6 (black) and 18 (yellow) $\mathrm{mJy} / \mathrm{beam}$. The white ellipse shows the region used to extract the XMM-Newton detected diffuse source.

than $3 \mathrm{mJy} /$ beam. This radio excess, with a $1 \sigma$ semi-axes of $35^{\prime \prime} \times 26^{\prime \prime}$, corresponds well to the position and size of the extended X-ray source. Integrating this excess yields a total flux density of the order of $19 \mathrm{mJy}$. A more conservative estimate of the flux density, based on the detection threshold of the MGPS-2 survey yields to an upper limit of $25 \mathrm{mJy}$.

We also searched the Parkes $2.4 \mathrm{GHz}$ survey of the southern galactic plane (Duncan et al. 1995) performed with the Parkes radio telescope for a possible counterpart. As the resolution of the Parkes image is only $10.4^{\prime}$ and the root-mean-square noise is approximately $12 \mathrm{mJy} /$ beam, we could only extract an upper limit of $\sim 100 \mathrm{mJy}$ at $2.4 \mathrm{GHz}$ for the extension of the diffuse $\mathrm{X}$-ray source.

\section{Searching the infrared band using Spitzer}

Moving towards the infrared band we analyzed the GLIMPSE and MIPSGAL surveys from the Spitzer Space Telescope.

\subsection{GLIMPSE}

The Galactic Legacy Infrared Mid-Plane Survey Extraordinaire (GLIMPSE, Benjamin et al. 2003) was performed with the IRAC instrument (Fazio et al. 2004) onboard Spitzer, which can simultaneously measure in four wavelengths at 3.6, 4.5, 5.8, and $8 \mu \mathrm{m}$. The final mosaic images have a resolution of $\sim 1.2^{\prime \prime}$. The $5 \sigma$ point source sensitivities are $0.2,0.2,0.4$ and $0.4 \mathrm{mJy}$ for the four different wavelengths, respectively.

We did not find any sign for diffuse infrared emission corresponding to the X-ray and radio counterparts. To determine upper limits we analyzed the infrared pixel flux distribution within and outside of the X-ray diffuse region, focusing on the 3.6 and $4.5 \mu \mathrm{m}$ images.

We selected all GLIMPSE pixels inside the X-ray source ellipse and subtracted the contribution of the brightest point-like sources. The final distribution was fitted with a Gaussian and compared with these obtained from four other similarly shaped ellipse regions extracted in the neighborhood. The average background flux obtained from the flux distributions varies among the various ellipses indicating a gradient of infrared emission in the region. The uncertainty on the average background flux determination $(\leq 0.04 \mathrm{MJy} / \mathrm{sr})$ integrated over the ellipse area represents the minimum flux density necessary to distinguish an additional diffuse emission with respect to the other regions. For a $3 \sigma$ detection limit, our upper limit becomes $\sim 25 \mathrm{mJy}$.

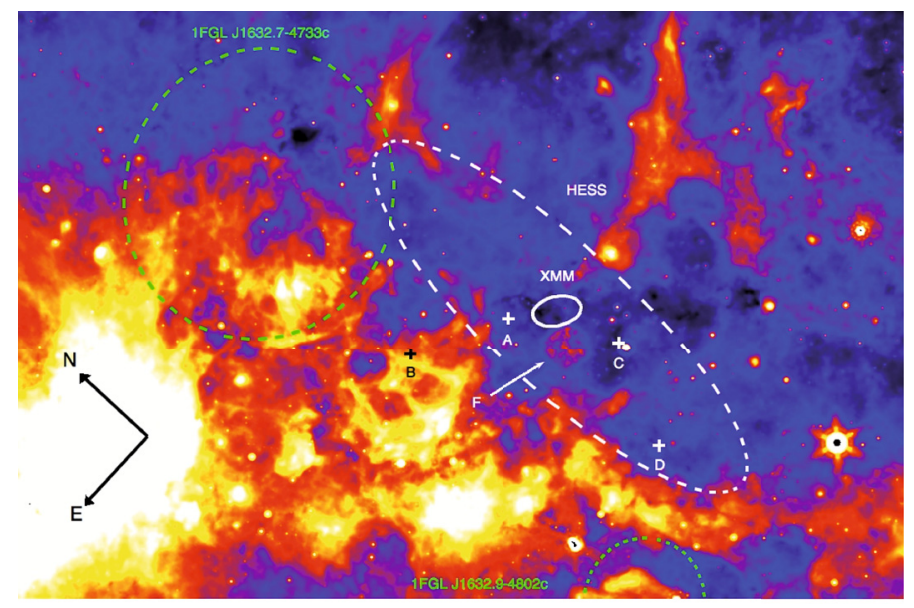

Fig. 4. An extraction from the MIPSGAL survey at $24 \mu \mathrm{m}\left(45^{\prime} \times 30^{\prime}\right)$. The small ellipse shows the position of the diffuse X-ray source, the dashed ellipse shows the HESS source extension (as in Fig. 11 of Aharonian et al. 2006a) and the two large dashed circles indicates the 95\% confidence region for the FERMI detections. The crosses indicate (A) XMMU J163219.9-474731, (B) AXJ1632.8-4746, (C) IGR $\mathrm{J} 16320-4751$, (D) the nearby radio pulsar PSR J1632-4757 and (F) the unidentified $24 \mu \mathrm{m}$ circular structure.

\subsection{MIPSGAL}

MIPSGAL (Carey et al. 2009) is a survey of the inner Galactic Plane using the Multiband Infrared Photometer for Spitzer (MIPS, Rieke et al. 2004). The survey field was imaged in two passbands, 24 and $70 \mu \mathrm{m}$ with a resolution of $6^{\prime \prime}$ and $18^{\prime \prime}$, and an estimated point source sensitivities $(3 \sigma)$ of 2 and $75 \mathrm{mJy}$, respectively. As for the GLIMPSE survey, there is no evidence for a diffuse emission corresponding to the diffuse X-ray source. In addition, at this wavelength, the infrared background is strongly inhomogeneous and does not allow to extract a useful upper limit.

Figure 4 displays the $24 \mu \mathrm{m}$ MIPSGAL mosaic with the various point sources, diffuse X-ray source and error circles mentioned in the text. It is worth noting that an unidentified circular structure (pointed by the "F" arrow), with a radius of $\sim 66$ ", visible at $24 \mu \mathrm{m}$ and not in the other bands, lies less than 93" away from the diffuse X-ray source. This object was detected by Flagey et al. (2009) but they have not found any counterpart. The nature of this object is unclear but its "monochromatic" spectrum does not favor non thermal emission and a Supernova remnant origin. In the 70 and $160 \mu \mathrm{m}$ there is no evidence for any emission coming from the extended X-ray source nor from the $24 \mu \mathrm{m}$ circular structure (source "F").

It is also interesting to remark that the extension of the $\mathrm{TeV}$ source lies in a region of faint emissivity at $24 \mu \mathrm{m}$ (Fig. 4). Perhaps this infrared cavity/edge corresponds to dust blown/heated by the progenitor supernova or high-energy photons.

\section{The GeV sources in the vicinity}

We looked for possible high energy counterpart in the FermiLAT first year Catalogue (Abdo et al. 2010), and found two unidentified GeV sources in the neighborhood of HESS J1632478 (see Fig. 4). Both these sources are flagged with the letter "c", indicating that they are to be considered as potentially confused with interstellar diffuse emission or perhaps spurious detection. Their location, flux and spectrum may not be reliable. 
1FGL J1632.9-4802c and 1FGL J1632.7-4733c lie 15.5' and respectively $16.3^{\prime}$ far from the extended source detected by XMM-Newton. As reported in Abdo et al. (2010), the diffuse background model in this region of the galaxy needs to be improved and the position of 1FGL J1632.7-4733c varies with that model. It is therefore very unclear if any of these Fermi sources could be a counterpart of HESS J1632-478. The HESS error ellipse is parallel to the line joining the two Fermi sources, lying close to the extremity of that ellipse. If the two Fermi sources are real and radiate in the TeVs, the extension and inclination of HESS J1632-478 could be significantly affected by confusion.

\section{Discussion}

\subsection{Spectral energy distribution}

The match in position and size of the radio excess and of the extended X-ray source, suggests a non thermal synchrotron source emitting from the radio to the X-rays. The TeV centroid of the HESS source lies within the extended source detected by XMM-Newton. All other X-ray sources are further away and point-like. This positional match and the fact that the TeV source is several times larger than the X-ray source, suggest that the non thermal synchrotron source also emits the VHE emission through inverse Compton processes.

The spectral energy distribution of the source, constructed with the HESS and XMM-Newton spectra, infrared upper limits and radio detection or upper limits, is featured on Fig. 5. The significant $\mathrm{GeV}$ fluxes extracted from the Fermi catalogue (Abdo et al. 2010) for the close-by Fermi source FGL J1632.7-4733c are also indicated. Together, the XMM-Newton and HESS spectra clearly indicate the presence of two spectral bumps matching the expected synchrotron and inverse Compton emission of a Pulsar Wind Nebula.

If $d_{3}$ is the distance of the source in unit of $3 \mathrm{kpc}$, the luminosities of the X-ray extended and point sources are $\sim 4 \times 10^{32} d_{3}^{2}$ and $2 \times 10^{32} d_{3}^{2} \mathrm{erg} / \mathrm{s}$, respectively. The bolometric luminosity of the synchrotron and inverse Compton components are $10^{34} d_{3}^{2}$ and $10^{35} d_{3}^{2} \mathrm{erg} / \mathrm{s}$, respectively, assuming the spectrum obtained in Fig. 5. Using the empirical relationship of Possenti et al. (2002), which presents a high dispersion, the X-ray luminosity is as expected for a pulsar with a total spin down power of $L_{\mathrm{SD}} \sim 10^{36} d_{3}^{1.5} \mathrm{erg} / \mathrm{s}$. The ratio of the gamma-ray and X-ray fluxes also suggest a pulsar spin-down power of $\sim 3 \times 10^{36} \mathrm{erg} / \mathrm{s}$ and an age of $\sim 2 \times 10^{4}$ years (Mattana et al. 2009). With $10 \%$ of the current spin down luminosity radiated at very high energies, HESS J1632-478 is among the oldest and most gamma-ray loud known pulsar wind nebulae (Kargaltsev et al. 2007), similar as these detected in HESS J1825-137 (Pavlov et al. 2008) or HESS J1640-465 (Lemiere et al. 2009).

Although not well defined, the $\mathrm{TeV}$ extension of the pulsar wind nebula is several times larger than the extension of the X-ray nebula, as observed for several aging pulsar wind nebula (HESS J1825-135, HESS J1420-607, HESS J1640-465; Aharonian et al. 2006b,c; Lemiere et al. 2009). This is usually explained by the much shorter lifetime of the electrons emitting synchrotron in the X-ray band compared to that of the electrons emitting inverse Compton in the TeVs. The very high energy synchrotron emitting electrons indeed do not have the time to reach the outer parts of the nebula. In the case of HESS J1632478 , the TeV extension is larger than the X-ray one, as observed in other sources. Note that the value of the ratio between the two components extensions can not be well constrained due to the

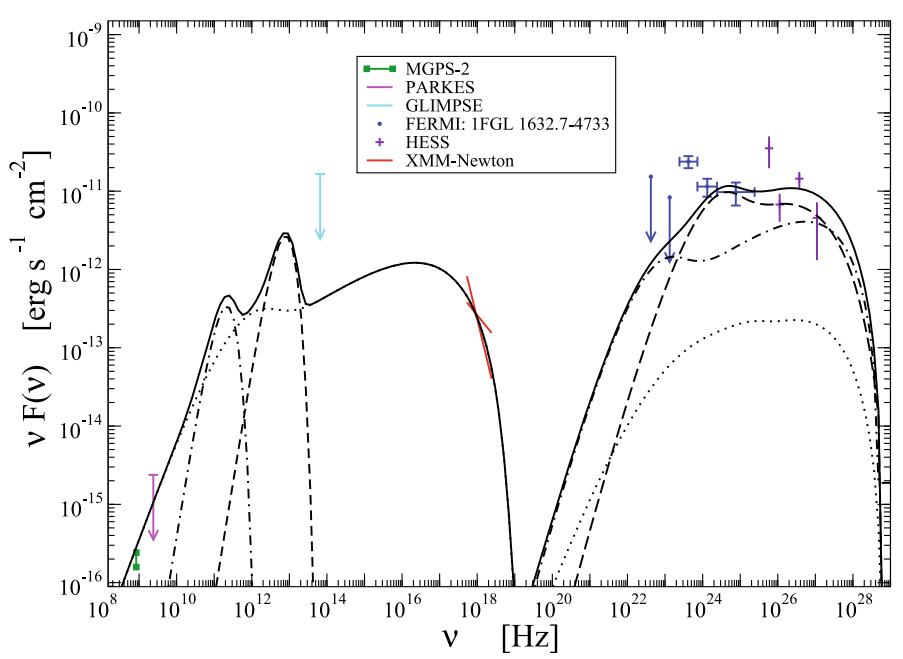

Fig. 5. Spectral energy distribution of HESS J1632-478, including the upper limits from PARKES (magenta) and GLIMPSE (cyan), the probable detection from MGPS-2(green), and the detections by XMM-Newton (red) and HESS (purple). The Fermi spectrum of the nearby source 1FGL 1632.7-4733 is also shown (in blue). The continuous line indicates the prediction of the model used to represent the emission. At low energy the dotted line represents the synchrotron emission from the electron distribution described by Eq. (5), the dotted-dashed and the dashed lines show the CMB and IR dust photons components respectively. At high energy, the dotted line represents the SSC component, the dotted-dashed and the dashed lines show the external Compton emission on the CMB and dust photons components respectively.

low $\mathrm{TeV}$ spatial resolution and that a better determination could be achieved when more sensitive/higher resolution Cherenkov instruments will become available and possible confusion with the other high energy sources detected in the field with Fermi (see Fig. 4) could be resolved.

\subsection{Nature of the emission}

An upper limit to the energy density of the synchrotron photons in the nebula can be estimated, using the peak of the maximum synchrotron emission compatible with the observed spectral energy distribution $\left(F_{\text {sync }}<10^{-10} \mathrm{erg} \mathrm{s}^{-1} \mathrm{~cm}^{-2}\right)$ and a lower limit of the angular size of the nebula $\left(\varnothing \sim 2^{\prime}\right)$, as

$U_{\mathrm{sync}}=\frac{L_{\mathrm{sync}}}{4 \pi c R^{2}}=\frac{F_{\mathrm{sync}}}{c \tan ^{2}(\varnothing / 2)}<0.02 \mathrm{eV} / \mathrm{cm}^{3}$,

where $R$ is the physical size of the nebula. This energy density is negligible when compared to that of the $\mathrm{CMB}\left(U_{\mathrm{CMB}}=\right.$ $\left.0.25 \mathrm{eV} / \mathrm{cm}^{3}\right)$ or that of the infrared galactic background in the galactic plane $\left(U_{\mathrm{rad}} \approx\right.$ few eV/cm ${ }^{3}$; Moskalenko et al. 2006) which will dominate the seed photons of the Inverse Compton component.

Assuming that the same electron population is responsible for the synchrotron and for the inverse Compton emission, the ratio of the two components luminosities is given by the ratio of the magnetic and radiation energy densities

$\frac{L_{\mathrm{sync}}}{L_{\mathrm{IC}}}=\frac{B^{2} / 2 \mu_{0}}{U_{\mathrm{rad}}}$,

thus the magnetic field in the nebula can be estimated as

$B=\left(2 \mu_{0} U_{\mathrm{rad}} \frac{F_{\mathrm{sync}}}{F_{\mathrm{IC}}}\right)^{0.5}=\sqrt{F_{\text {sync }} / F_{\mathrm{IC}}} \times 10 \mu \mathrm{G}$. 
As the spectral energy distribution qualitatively indicates $L_{\text {sync }}<$ $L_{\mathrm{IC}} / 10$ (considering the highest energy electrons, in the synchrotron nebula), the average magnetic field in the nebula is $B \sim 3 \mu \mathrm{G}$. The spectral energy distribution also indicates that the cutoff of the synchrotron emission is in the range $10^{16-18} \mathrm{~Hz}$. The energy of the electrons emitting at that maximum is in the range

$\gamma_{\max }=\left(\frac{2 \pi m_{\mathrm{e}} c v_{\mathrm{sync}}}{e B}\right)^{0.5}=(1-10) \times 10^{7}$.

The maximum of the inverse Compton emission is therefore expected at $\sim(1-10) \times 10^{27} \mathrm{~Hz}$, in agreement with the $\mathrm{TeV}$ spectral energy distribution.

A possible phenomenological description of the electron energy distribution is given by the model recently presented by Spitkovsky (2008). This author, starting from a numerical twodimensional particle in cell simulation, found a spectrum that, at the downstream of the shock front, can be described by a relativistic Maxwellian plus a power-law cut-off high energy tail. Such a model has been recently adopted by Fang \& Zhang (2010) to model the broad band SED of a sample of PWNs. We adopt the same analytical model that reads:

$n(\gamma)= \begin{cases}K\left(\frac{\gamma}{\gamma_{c}}\right) \exp \left(-\frac{\gamma}{\gamma_{c}}\right)=n_{0}(\gamma) & \gamma \leq \gamma_{1} \\ n_{0}(\gamma)+K_{1}\left(\frac{\gamma}{\gamma_{c}}\right)^{-\alpha} \exp \left(-\frac{\gamma}{\gamma_{c 1}}\right) & \gamma>\gamma_{1},\end{cases}$

where $\gamma_{\mathrm{c}}$ is the typical Lorentz factor of the electrons at the termination shock, $\alpha$ is the spectral index of the high-energy tail, with a cut-off Lorentz factor $\gamma_{\mathrm{c} 1}$. We fix $\gamma_{1}=7 \gamma_{\mathrm{c}}$ according to Spitkovsky (2008) and $K_{1}$ is normalized in order to match the Maxwellian and the high-energy tail.

We set the Lorentz factor for the particles at the termination shock to $\gamma_{\mathrm{c}}=1.6 \times 10^{5}$, and use the same value of $\alpha=2.4$ as obtained in Spitkovsky (2008). In order to match the X-ray data we use $\gamma_{\mathrm{c} 1}=1.6 \times 10^{8}$. The resulting spectral energy distribution, reproduced using a well tested code (Tramacere 2007; Tramacere et al. 2009) is reported in Fig. 5 together with the observed data. We reproduce both the synchrotron self Compton (SSC) and the external Compton emission, assuming a one-zone (post terminal shock) homogeneous emitting region. The details of the best-fit model parameters are reported in Table 4. These parameters are only representative and a simpler model could be fitted to the data as well. The quality and the uncertainties on the high energy data are such that deriving accurate information on the electron distribution is not possible.

Although the Fermi data are partly compatible with the emission model, it is difficult to say if the soft GeV spectrum of 1FGL $1632.7-4733 \mathrm{c}$ is related to the pulsar wind nebula or should be considered as upper limits. Indeed, the HESS/XMM source lies well outside of the $95 \%$ confidence region of 1FGL 1632.74733c (Fig. 4).

With the shape of the electron distribution inferred from the external Inverse Compton component, the strength of the synchrotron component is fairly well constrained by the X-ray detection and by the radio detection/upper limits.

The total energy in the electron distribution amounts to $10^{48} \mathrm{erg}$. This energy is comparable to the product of a spindown pulsar power at birth of $10^{38} \mathrm{erg} / \mathrm{s}$ and of a characteristic decay time of hundreds of years.

The nebula magnetic field, size, luminosity and inferred age are in reasonable agreement with the simulations by Fang \& Zhang (2010).
Table 4. Emission model representing the spectral energy distribution of HESS J1632-478 (assuming a distance of $3 \mathrm{kpc}$ ).

\begin{tabular}{llrl}
\hline \hline & Parameter & Value & \\
\hline Electron distribution & $\gamma_{\mathrm{c}}$ & $1.6 \times 10^{5}$ & \\
& $\gamma_{\mathrm{c} 1}$ & $1.6 \times 10^{8}$ & \\
& $\alpha$ & 2.4 & \\
& $N$ & $7.0 \times 10^{-8}$ & $\mathrm{e}^{-} / \mathrm{cm}^{3}$ \\
Synchrotron & $R$ & $1.6 \times 10^{18}$ & $\mathrm{~cm}$ \\
External Compton & $B$ & 1 & $\mu \mathrm{G}$ \\
& $U_{\text {rad }}^{\mathrm{CMB}}$ & 0.25 & $\mathrm{eV} / \mathrm{cm}^{3}$ \\
& $U_{\mathrm{rad}}^{\mathrm{R}}$ & 2 & $\mathrm{eV} / \mathrm{cm}^{3}$ \\
\hline
\end{tabular}

\subsection{Absorption and distance}

The absorbing column densities measured by XMM-Newton in the field are much in excess to the values $N_{\mathrm{H}_{\text {gal }}}=(1.5-1.8) \times$ $10^{22} \mathrm{~cm}^{-2}$ derived from the low resolution radio surveys (Kalberla et al. 2005; Dickey \& Lockman 1990).

The absorbing column density on the pulsar wind nebula $\left(N_{\mathrm{H}} \approx 11 \times 10^{22} \mathrm{~cm}^{-2}\right)$ is very similar to that observed for IGR J16320-4751 and slightly larger than these observed in the directions of AX J1632.8-4746 and XMMU J163219.9-474731 $\left(N_{\mathrm{H}} \approx 6 \times 10^{22} \mathrm{~cm}^{-2}\right)$. This indicates an unusually large column density in this field and that about half of the absorption towards IGR J16320-4751 (Walter et al. 2006) may not be related to the High-Mass X-Ray Binary system. It also indicates that the distance to HESS J1632-478 is of the same order as that of IGR J16320-4751, which was approximately estimated as $3.5 \mathrm{kpc}$ (Rahoui et al. 2008).

\section{Conclusions}

We observed the unidentified TeV source HESS J1632-478 with $X M M-N e w t o n$ and looked for counterparts in the $\mathrm{GeV}$, infrared and radio bands. An extended faint X-ray source is detected close to the centroid of the HESS error ellipse. A radio excess corresponding to the X-ray source is found in the Molonglo sky survey. Upper limits have been derived from Spitzer and Parkes data. The GeV image obtained by Fermi shows two close-by sources flagged as confused in the Fermi catalogue, but none of them corresponds to the X-ray source, the situation is therefore unclear.

The flux density emitted by HESS J1632-478 at very high energies is at least 20 times larger than observed at the other wavebands probed. The source shape and spectral energy distribution suggests a pulsar wind nebula and can be used to successfully constrain a one zone model for the post terminal shock region of the pulsar wind nebula. The assumed relativistic electron distribution is Maxwellian $\left(\gamma \sim 10^{5}\right)$ with a non thermal tail extending to $\gamma \sim 10^{8}$. The synchrotron nebula is faint because of the low magnetic field $(3 \mu \mathrm{G})$.

The point-like X-ray source, detected in the synchrotron nebula, is probably the signature of the pulsar and of the termination shock. The age of the pulsar is estimated as some $10^{4}$ years. The lack of spatial resolution and the probable confusion of the $\mathrm{GeV} / \mathrm{TeV}$ sources in this field does not allow to perform a detailed study of the inverse Compton emitting region and of its interactions. More sensitivity and spatial resolution are needed at very high energies.

Acknowledgements. Based on observations obtained with XMM-Newton, an ESA science mission with instruments and contributions directly funded by ESA Member States and NASA. The Molonglo survey is supported by the 
M. Balbo et al.: HESS J1632-478: an energetic relic

Australian Research Council. J.A.Z.H. acknowledges the Swiss National Science Foundation for financial support.

\section{References}

Abdo, A. A., Ackermann, M., Ajello, M., et al. 2010, ApJS, 188, 405 Aharonian, F., Akhperjanian, A. G., Bazer-Bachi, A. R., et al. 2006a, ApJ, 636, 777

Aharonian, F., Akhperjanian, A. G., Bazer-Bachi, A. R., et al. 2006b, A\&A, 460, 365

Aharonian, F., Akhperjanian, A. G., Bazer-Bachi, A. R., et al. 2006c, A\&A, 456, 245

Benjamin, R. A., Churchwell, E., Babler, B. L., et al. 2003, PASP, 115, 953

Carey, S. J., Noriega-Crespo, A., Mizuno, D. R., et al. 2009, PASP, 121, 76

Cassinelli, J. P., Waldron, W. L., Sanders, W. T., et al. 1981, ApJ, 250, 677

Dickey, J. M., \& Lockman, F. J. 1990, ARA\&A, 28, 215

Duncan, A. R., Stewart, R. T., Haynes, R. F., \& Jones, K. L. 1995, MNRAS, 277, 36

Fang, J., \& Zhang, L. 2010, A\&A, 515, A20

Fazio, G. G., Hora, J. L., Allen, L. E., et al. 2004, ApJS, 154, 10

Flagey, N., Billot, N., Carey, S., et al. 2009, in Am. Astron. Soc. Meet. Abstracts, $214,316.04$
Hinton, J. A. 2004, New Astron. Rev., 48, 331

Jansen, F., Lumb, D., Altieri, B., et al. 2001, A\&A, 365, L1

Kaastra, J. S. 1992, in Internal SRON-Leiden Report, updated version 2.0

Kalberla, P. M. W., Burton, W. B., Hartmann, D., et al. 2005, A\&A, 440, 775

Kargaltsev, O., Pavlov, G. G., \& Garmire, G. P. 2007, ApJ, 660, 1413

Lemiere, A., Slane, P., Gaensler, B. M., \& Murray, S. 2009, ApJ, 706, 1269

Mattana, F., Falanga, M., Götz, D., et al. 2009, ApJ, 694, 12

Moskalenko, I. V., Porter, T. A., \& Strong, A. W. 2006, ApJ, 640, L155

Murphy, T., Mauch, T., Green, A., et al. 2007, MNRAS, 382, 382

Pavlov, G. G., Kargaltsev, O., \& Brisken, W. F. 2008, ApJ, 675, 683

Possenti, A., Cerutti, R., Colpi, M., \& Mereghetti, S. 2002, A\&A, 387, 993

Rahoui, F., Chaty, S., Lagage, P., \& Pantin, E. 2008, A\&A, 484, 801

Rieke, G. H., Young, E. T., Engelbracht, C. W., et al. 2004, ApJS, 154, 25

Spitkovsky, A. 2008, ApJ, 682, L5

Strüder, L., Briel, U., Dennerl, K., et al. 2001, A\&A, 365, L18

Sugizaki, M., Mitsuda, K., Kaneda, H., et al. 2001, ApJS, 134, 77

Tramacere, A. 2007, Ph.D. Thesis, Spectral Variability in Blazar's High Energy Emission La Sapienza University, Rome (2007)

Tramacere, A., Giommi, P., Perri, M., Verrecchia, F., \& Tosti, G. 2009, A\&A, 501,879

Turner, M. J. L., Abbey, A., Arnaud, M., et al. 2001, A\&A, 365, L27

Walter, R. 2007, Ap\&SS, 309, 5

Walter, R., Zurita Heras, J., Bassani, L., et al. 2006, A\&A, 453, 133 